truly chaotic behaviour, has been tempered by a recent calculation ${ }^{8}$ which indicates that a plausible model of the $\mathrm{BZ}$ reaction can indeed generate chaos under homogeneous conditions. It now seems likely that chaos in chemical systems may have two sources - complex homogeneous kinetics and/or interactions between kinetics and mixing.

The work of Menzinger and Dutt ${ }^{1}$ is unlikely to be the last word on the nature of mixing effects in nonlinear chemical kinetics. The $0.25-\mathrm{mm}$ microelectrode that they use to monitor spatial and temporal variations in electrochemical potential gives only very crude spatial resolution; a recent study of pattern formation in the $\mathrm{BZ}$ reaction" uses electrodes with micrometre dimensions. By monitoring simultaneously two micro-

electrodes at different locations in the reactor, Menzinger and Dutt find that when the reactor switches from one steady state to another, both the microelectrodes undergo a change in potential at the same time, even though their absolute potentials are quite different. On the other hand, certain chemical systems in a closed reactor show evidence of a transition between stable states that nucleates locally and then propagates through the system ${ }^{10}$. But although there are clearly questions still to be resolved, chemists are growing increasingly aware of the danger of assuming that they ever conduct their reactions in media of uniform concentration.

Irving R. Epstein is Helena Rubenstein Professor of Chemistry at Brandeis University, Waltham, Massachusetts 02254-9110, USA.

\section{Tamarin compatibility}

\section{Shirley A. Ellis and Andrew J. McMichael}

OVEr the past few years it has emerged that the major histocompatibility complex (MHC) class I region of human chromosome 6 contains expressed genes in addition to the (so-called classical) A, B and $\mathrm{C}$ loci'. The classical class I genes encode highly polymorphic transmembrane glycoproteins, whose main role is to present peptides from processed foreign antigen to cytotoxic $\mathrm{T}$ lymphocytes. To date, three other distinct loci (E, F and G) have been identified ${ }^{2}$ and restriction fragment length polymorphisms suggest that there will be others. The origin and function of these genes have, however, proved elusive. On page 60 of this issue ${ }^{3}$, Watkins et al. provide insights into the possible function of the products of one of these loci, HLA G, from a surprising source - the cotton-top tamarin.

The authors cloned and sequenced all of the MHC class I genes from a sample of 79 unrelated members of this species, and demonstrate that only 11 molecules are expressed. When these were compared with each other, they showed far less variability in amino-acid sequence than is seen between alleles of the class I loci in either man or mouse; ten out of the 11 molecules are more closely related to human HLA G than to classical A, B and $\mathrm{C}$ molecules. We must assume that these 'G-like' molecules are functional in the cotton-top tamarin, because the animals can mount cytotoxic T-cell responses. But when they encounter pathogens not found in their natural environment (EpsteinBarr, Rous sarcoma, measles and parainfluenza viruses, for example), they prove to be particularly vulnerable, a phenomenon which may be related to their lack of class I polymorphism.

We cannot assume however that because the cotton-top tamarin MHC class I appears to function normally, albeit similar role. There are several differences to be considered. The two full-length human $\mathrm{G}$ (derived) amino-acid sequences that have been reported ${ }^{4.5}$ are identical; together with isoelectric focusing studies ${ }^{6.7}$ this shows that there is probably no polymorphism (at the amino-acid level) at the $\mathrm{G}$ locus. The only cells that have been demonstrated to express the HLA G product are extravillous cytotrophoblast from the placenta and some trophoblastderived choriocarcinoma cell lines. Thus the HLA G molecule is found only on cells that do not express HLA class I A, B or C. Possibly the most striking feature of HLA $G$ which really sets it apart from classical HLA class I is its truncated cytoplasmic tail - the shortened tail is due to a termination codon at the beginning of exon 6 (the first cytoplasmic domain) that results in a smaller expressed product of relative molecular mass $\left(M_{\mathrm{r}}\right) \quad 39,000$. Although it does appear to be anchored in the cell membrane, this missing portion may have implications for the function of the molecule. The 'HLA G-like' molecules seen on the tamarin cells have a normal cytoplasmic tail, and are the size of classical class I molecules, $M_{\mathrm{r}} 43,000$.

The human placental trophoblast cells, which are located at the maternal-fetal interface, are thought to play a part in the survival of the semiallogeneic fetus. It was believed that these cells formed an MHC-negative 'barrier' against maternal immune surveillance ${ }^{8}$, but recent work has shown that they express high levels of HLA G (refs 5, 7). The function of this molecule is not known - it could be nonimmunological, related to the invasive in a limited way, the human HLA G has a properties of this tissue, or to the effects of peptide hormones. It may be necessary to have a 'neutral' HLA class I molecule, having a passive but important role, for example, to resist attack by natural killer cells. Selection pressure against polymorphism of an HLA molecule expressed at this extremely sensitive site seems likely, and implies a function fundamentally different from that of the other HLA class I antigens, where a high level of polymorphism plays a crucial part in their immunological activities.

These results raise questions regarding the original nature and role of the ancestral HLA $G$ genes. If the HLA G-like gene products functioned as classical class I molecules in the primate ancestor, they would have needed to 'lose' their polymorphism in adapting to a role in the trophoblast. Alternatively, the ancestral $\mathrm{G}$ genes might never have functioned as classical class I molecules; in the tamarin ancestor, accidental deletion of the classical loci may have resulted in an increase in polymorphism at the $G$ locus and a wider tissue distribution. As Watkins et al. point out, that these primates are born as stable, bone-marrowchimaeric twins may be relevant. This might help to compensate for the negative effect of the polymorphism of class I molecules expressed on the trophoblast; females might be tolerant of other parental haplotypes and would therefore be less likely to reject a fetus that carried a foreign MHC class I type.

We need to know therefore if the trophoblast cells in the tamarin express the same class I products as other tissues, or if they show limited or quite different expression. It will also be worthwhile to look more closely at other species that show limited MHC class I polymorphism, perhaps for different reasons (for example the cheetah ${ }^{10}$ and the Syrian hamster ${ }^{11}$ ), to see if there too evolutionary pressures have acted in a similar way.

Shirley A. Ellis and Andrew J. McMichael are in the Institute of Molecular Medicine, John Radcliffe Hospital, Oxford OX3 9DU, UK.

1. Orr, H.T., Koller, B.H., Geraghty, D. \& DeMars, R. in Evolution and Vertebrate Immunity (eds Kelsoe, G. and Schulze, D.H.) (University of Texas Press, 1987)

2. Shimizu, Y., Geraghty, D.E., Koller, B.H., Orr, H.T. \& DeMars, R. Proc. natn. Acad. Sci. U.S.A. 227-231 (1988).

3. Watkins, D.I. et al. Nature 346, 60-63 (1990).

4. Geraghty, D.E., Koller, B.H. \& Orr. H.T. Proc. natn. Acad Sci. U.S.A. 84, 945-949 (1987).

5. Ellis, S.A., Palmer, M.S. \& McMichael, A.J. J. Immun 144, 731-735 (1990).

6. Ellis, S.A., Sargent, I.L., Redman, C.W.G. \& MCMichael, A.J. Immunology 59, 595-601 (1986).

7. Kovats, S. et al. Science 248, 220-223 (1990).

8. Head, J.R. \& Billingham, R.E. in Immunological Aspects of Reproduction (ed Crighton. D.B.) 133-152 (Butterworths, London, 1984)

9. Storkus, W.J., Alexander, J., Payne, J.A., Dawson, J.R. \& Cresswell, P. Proc. natn. Acad. Sci. U.S.A. 2361-2364 (1989).

10. O'Brien, S.J. et al. Science 277, 1428-1431 (1985)

11. Darden, A.G. \& Streilen, J.W. Immunogenetics 20,603607 (1984) 